\title{
Insight in the 3D morphology of silica-based nanotubes using electron microscopy
}

Teresa Dennenwaldt ${ }^{* \#}$, Andreas Wisnet ${ }^{2}$, Stefan J. Sedlmaier ${ }^{3}$, Markus Döblinger $^{2}$, Wolfgang Schnick ${ }^{2}$, Christina Scheu ${ }^{1}$

${ }^{1}$ Max-Planck-Institut für Eisenforschung GmbH, Max-Planck-Straße 1, 40237 Düsseldorf, Germany

${ }^{2}$ Department of Chemistry, Ludwig-Maximilians-Universität München, Butenandtstraße 5-13, 81377 Munich, Germany

${ }^{3}$ Karlsruhe Institute of Technology, Institute of Nanotechnology, Hermann-von-Helmholtz-Platz, 1, 76344 EggensteinLeopoldshafen, Germany

*new address: Electron Spectrometry and Microscopy Laboratory and Interdisciplinary Center for Electron Microscopy, École Polytechnique Fédérale de Lausanne, EPFL SB ICMP LSME, MXC 135, Station 12,1015 Lausanne, Switzerland

${ }^{\#}$ Corresponding author: Dr. Teresa Dennenwaldt, +41216936813,teresa.dennenwaldt@epfl.ch

Keywords: nanotubes, template-free synthesis, tuning, electron tomography

\begin{abstract}
Amorphous silica-based nanotubes (SBNTs) were synthesized from phosphoryl triamide, $\mathrm{OP}\left(\mathrm{NH}_{2}\right)_{3}$, thiophosphoryl triamide, $\mathrm{SP}\left(\mathrm{NH}_{2}\right)_{3}$, and silicon tetrachloride, $\mathrm{SiCl}_{4}$, at different temperatures and with varying amount of the starting material $\mathrm{SiCl}_{4}$ using a recently developed template-free synthesis approach. Diameter and length of the SBNTs are tunable by varying the synthesis parameters. The 3D mesocrystals of the SBNTs were analyzed with focused ion beam sectioning and electron tomography in the transmission electron microscope showing the hollow tubular structure of the SBNTs. The reconstruction of a small SBNT assembly was achieved from a high-angle annular-dark field scanning transmission electron microscopy tilt series containing only thirteen images allowing analyzing beam sensitive material without altering the structure. The reconstruction revealed that the individual nanotubes are forming an interconnected array with an open channel structure.
\end{abstract}

\section{Introduction}

One dimensional (1D) inorganic nanomaterials like nanowires (NWs), nanotubes (NTs) or -rods exhibit fascinating properties such as efficient transport of electrons and optical excitations and are therefore promising for a variety of applications. Different approaches do exist to synthesize and grow those nanostructures which can further be directed into two dimensional (2D) or even threedimensional (3D) structures. This is essential for nowadays science and technology (Agrawal et al., 2015; Betzler et al., 2014; García-Calzón and Díaz-García, 2012; Joshi and Schneider, 2012; Yang et al., 2011). 1D nanostructures, for instance, are used as building blocks for applications like sensors, electronics, and photonics or as nanofillers in composites (Fan et al., 2015; Joshi and Schneider, 2012; Ni et al., 2015). Their properties are based on several specific parameters. They possess a very high surface-to volume ratio due to their length and diameter dimensions (Bréchignac et al., 2007). Nanomaterials offer a range of new applications. Various growth methods allow tailoring diverse 1D 
nanostructures (e.g carbon NTs, metal oxide NWs and NTs, III-V or II-VI based heteronanostructures and metallic NWs) (Bréchignac et al., 2007; Joshi and Schneider, 2012) and their 2D or 3D architectures for respective applications. The most frequently applied synthesis methods for NTs employs structure-directing templates (Bréchignac et al., 2007; García-Calzón and Díaz-García, 2012; Rao and Govindaraj, 2009; Yang et al., 2011). This includes inorganic templates like porous alumina membranes or inorganic nanomaterials (nanowires, -rods, or -tubes), organic self-assembled gels or biological and biomolecular templates like proteins and peptides (Fan et al., 2015; García-Calzón and Díaz-García, 2012; Rao and Govindaraj, 2009; Yang et al., 2011). A benefit of template-based approaches is the control of diameter and length of the NTs and the great variety of synthesis routes, e. g. sol-gel, hydrothermal or microemulsion synthesis methods. After growth, the template must be removed by dissolution or calcination, which often cannot be achieved completely. The challenge is to liberate the NTs without damage or even full collapse. Furthermore, by removal of the template, the orientation or ordered arrangement of the NTs may be lost; the nanostructures may even collapse entirely. The importance of developing template-free approaches is revealed by a new method reported by Ren et al. (Ren et al., 2014). Silica fibers are synthesized using the centrifugal jet spinning technique. After spinning and annealing SNT fibers are obtained.

To design 2D or 3D structures control over dimensions of the nanomaterials is indispensable and investigation of prepared nanostructures require analyzing methods with high resolution. Transmission electron microscopy (TEM) and its analytical techniques allow an in-depth characterization at the nanoscale. Nevertheless, conventional TEM depicts a 2D projection of a 3D object. To overcome this problem of loss by 2D projections, series of TEM or scanning TEM (STEM) images at various tilt angles can be acquired and 3D reconstruction of an object can be achieved. A range of techniques is currently applied where weighted back-projection (WBP) and the iterative techniques like simultaneous iterative reconstruction technique (SIRT) are the most prominent ones (E. Biermans, L. Molina, K. J. Batenburg, S. Bals, 2010; Midgley and Dunin-Borkowski, 2009). STEM imaging is often used for electron tomography experiments as diffraction artifacts and phase contrast effects in TEM mode can hinder or mislead the interpretation of the data (Hyun et al., 2008). Electron tomography is a powerful tool to obtain insight in the full 3D morphology of micro- and nano-sized objects (Britt et al., 2013; E. Biermans, L. Molina, K. J. Batenburg, S. Bals, 2010; Midgley and DuninBorkowski, 2009). Not only the external morphology can be determined but also the inner structure of e.g. NTs can be made visible in 3D (Hungría et al., 2009; Suh et al., 2013).

In this paper, we report about controlling the diameter and length of template-free synthesized amorphous silica-based nanotubes (SBNTs) that build 3D mesocrystals by varying the synthesis conditions such as temperature and ratio of the starting materials. In the recently reported novel template-free approach for SBNTs phosphoryl triamide, $\mathrm{OP}\left(\mathrm{NH}_{2}\right)_{3}$, (Klement and Koch, 1954) thiophosphoryl triamide, $\mathrm{SP}\left(\mathrm{NH}_{2}\right)_{3},(\mathrm{Schnick}, 1989)$ and silicon tetrachloride, $\mathrm{SiCl}_{4}$, were mixed under inert gas atmosphere, sealed in silica glass ampoules and heated to different temperatures ranging from 
200 to $700{ }^{\circ} \mathrm{C}$ (Sedlmaier et al., 2012). We obtained the reaction products, flakes and NTs as well as hollow particles as side product, consisting all of $\mathrm{Si}, \mathrm{P}, \mathrm{O}$, and $\mathrm{N}$ which were amorphous in $\mathrm{X}$-ray and electron diffraction (Dennenwaldt et al., 2014; Sedlmaier et al., 2012). The SBNTs exhibit a bamboolike structure. With electron energy loss spectroscopy (EELS) in the TEM on the nanoscale we found that the bonding behavior of the elements and the electronic structure of the SBNTs is a mixture of silicon dioxide $\left(\mathrm{SiO}_{2}\right)$ and silicon nitride $\left(\mathrm{Si}_{3} \mathrm{~N}_{4}\right)$ (Dennenwaldt et al., 2014). Furthermore, the SBNTs show a much higher stability against bases (Dennenwaldt et al., 2014) compared to SNTs reported in the literature (Hu et al., 2010). In the present work, the 3D arrangement, the internal morphology, diameter and length of these SBNTs is elucidated with focused ion beam (FIB) microscopy, TEM and STEM-HAADF tomography and correlated to the synthesis conditions. In comparison to our earlier studies we synthesized SBNTs using a higher $\mathrm{SiCl}_{4}$ precursor concentration and contrast the results to the SBNTs synthesized with a lower $\mathrm{SiCl}_{4}$ precursor concentration.

\section{Materials and Methods}

\subsection{Scanning electron microscopy}

A JSM-6500F SEM (JEOL Ltd., Tokyo, Japan) with a field emission source operated at 4.0-12.0 kV equipped with an EDX detector model 7418 (Oxford Instruments, Oxfordshire, UK) was used to acquire SE images. For SEM sample preparation powder was placed on a cylindrical brass sample holder fixed with self-adhesive carbon plates (Plano, Wetzlar, Germany) and sputtered with carbon (sputter device: BAL-TEC MED 020, BAL-TEC AG, Balzers, Netherlands).

\subsection{Transmission electron microscopy}

For TEM sample preparation powder was suspended in ethanol and the solution was dropped on a lacey C-coated Cu grid (Lacey S166-2, PLANO). TEM studies were performed on a Titan 80-300 $\mathrm{keV} \mathrm{S/TEM} \mathrm{equipped} \mathrm{with} \mathrm{an} \mathrm{EDX} \mathrm{detector} \mathrm{and} \mathrm{an} \mathrm{energy} \mathrm{filter} \mathrm{for} \mathrm{EELS} \mathrm{measurements} \mathrm{and} \mathrm{on} \mathrm{a}$ $200 \mathrm{kV}$ FEI monochromated F20 UT Tecnai (STEM/TEM) located at the NCEM in Berkeley. The tilt series used for the reconstruction has been acquired at $80 \mathrm{keV}$ in STEM-HAADF mode using a tilt range of $\pm 70^{\circ}$ with steps of $10^{\circ}$. The inner diameter of the HAADF detector was $37 \mathrm{mrad}$, the outer diameter $200 \mathrm{mrad}$ and the convergence angle $16.7 \mathrm{mrad}$ using a condenser aperture of $50 \mu \mathrm{m}$.

\subsection{Focused ion beam microscopy}

A 3D assembly of the SBNTs was investigated by FIB sectioning on a Zeiss NVision40 FIB microscope. SE images were acquired with a low acceleration voltage of $2.5 \mathrm{kV}$. A carbon protection layer was deposited on the SBNT mesocrystal with successive ion beam induced deposition with a beam current of $300 \mathrm{pA}$ at $30 \mathrm{kV}$. Cutting and thinning were performed at $30 \mathrm{keV}$, the polishing 30 $\mathrm{keV}$ with a beam current of $30 \mathrm{pA}$ followed by $10 \mathrm{pA}$.

\subsection{Synthesis of the SBNTs}


According to our recently published work (Sedlmaier et al., 2012) the triamides $\mathrm{OP}\left(\mathrm{NH}_{2}\right)_{3}$ (Klement and Koch, 1954) (18.6 mg, $0.196 \mathrm{mmol})$ and $\mathrm{SP}\left(\mathrm{NH}_{2}\right)_{3}$ (Schnick, 1989) (70.0 mg, $\left.0.630 \mathrm{mmol}\right)$ were mixed and ground in an argon filled glove box and transferred into a flame-dried silica glass ampule (wall thickness $2 \mathrm{~mm}$, inner diameter $11 \mathrm{~mm}$ ). $\mathrm{SiCl}_{4}$ (Sigma-Aldrich, 99.998\%) was then dropped onto the mixture. For the low concentration $33.8 \mu \mathrm{L}(0.294 \mathrm{mmol})$ were added and for the high concentration $67.6 \mu \mathrm{L}(0.589 \mathrm{mmol})$ while all other parameters like heating rate and dwell time were kept constant. During the synthesis nitrogen was used as inert gas. After having cooled the ampoule with liquid nitrogen it was sealed (to a length of approximately $11 \mathrm{~cm}$ ) under reduced pressure and subsequently heated in a conventional tube furnace in horizontal position to $200{ }^{\circ} \mathrm{C}$ and different target temperatures $\left(300-700{ }^{\circ} \mathrm{C}\right.$ in steps of $100{ }^{\circ} \mathrm{C}$, dwell times of 12 and $48 \mathrm{~h}$, heating and cooling rate $1 \mathrm{~K} \mathrm{~min}^{-1}$ ). The reaction product was gained as a dry, gray solid.

\subsection{Algorithm and reconstruction}

After STEM-HAADF image acquisition the alignment was performed via ImageJ, (Rasband,19972011) using the TomoJ add-on (Messaoudi et al., 2007) which includes a landmark-based alignment script (Sorzano et al., 2009). A masked SIRT was used to obtain an adequate segmentation and a good starting model to achieve the actual morphological reconstruction. This step was accomplished by a discrete algebraic reconstruction technique step (Zürner et al., 2012). Thirteen images have been used for reconstruction of the SBNTs fragment.

\section{Results and discussion}

First experiments concerning the influence of the $\mathrm{SiCl}_{4}$ precursor concentration were made at $700{ }^{\circ} \mathrm{C}$ (Sedlmaier et al., 2012). Here, the amount of the Si source was increased while all other parameters (ratio of other starting materials, heating rate, temperature and dwell time) were kept constant. In the current work we performed a systematic study where we varied the temperature and the $\mathrm{SiCl}_{4}$ concentration, with the main goal to elucidate the effect of the synthesis parameters on the 3D network. In the following, the two investigated $\mathrm{SiCl}_{4}$ concentrations will be denominated as high and low $\mathrm{SiCl}_{4}$ precursor concentrations. Thereby, the high concentration corresponds to double as much primary concentration of $\mathrm{SiCl}_{4}$ (Sedlmaier et al., 2012). The SBNTs form uniformly hyperbranched 3D mesocrystals as can be seen in the scanning electron microscopy (SEM) image of a sample prepared at $700{ }^{\circ} \mathrm{C}$ with high $\mathrm{SiCl}_{4}$ precursor concentration (Figure 1a). The other reaction products like flakes and hollow particles are present as well. Figure 1b shows a bright-field TEM (BF-TEM) image of the SBNTs revealing the bamboo-like structure. The synthesis temperature was varied in steps of $100{ }^{\circ} \mathrm{C}$ in the range between 200 and $700{ }^{\circ} \mathrm{C}$. It could be found that all three reaction products appear at both $\mathrm{SiCl}_{4}$ precursor concentrations and at every temperature.

Independent from the synthesis temperature and $\mathrm{SiCl}_{4}$ precursor concentration, EDX and EELS analyses reveal that the SBNTs and the hollow particles are predominantly containing $\mathrm{Si}, \mathrm{O}$ and N, 
whereas the flakes have a higher amount of $\mathrm{P}$ and $\mathrm{N}$. This is in good agreement with our former study of SBNTs synthesized at a temperature of $700{ }^{\circ} \mathrm{C}$ and a low $\mathrm{SiCl}_{4}$ precursor concentration (Dennenwaldt et al., 2014; Sedlmaier et al., 2012). Analyzing the SBNTs synthesized at different temperatures with varying amounts of $\mathrm{SiCl}_{4}$ precursor, we found that the chemical composition averaged over all reaction products is very similar ( $\mathrm{Si}: \mathrm{O}: \mathrm{N}: \mathrm{P}=28: 40: 25: 3$ at.\%), independent from these two parameters. Also, like in previous studies the tip and walls of the SBNTs have the same chemical composition (Dennenwaldt et al., 2014; Sedlmaier et al., 2012). Electron energy-loss near-edge structure (ELNES) analysis of the Si-L $\mathrm{L}_{2,3}$-edge in the EELS spectra of the samples prepared with a high $\mathrm{SiCl}_{4}$ precursor concentration reveal that $\mathrm{Si}$ is predominantly tetrahedrally coordinated with $\mathrm{O}$ and $\mathrm{N}$. The ELNES data of the $\mathrm{Si}-\mathrm{L}_{2,3}$-edge are in good agreement with those synthesized with a low $\mathrm{SiCl}_{4}$ precursor content. An exemplary comparison of the Si- $\mathrm{L}_{2,3}$-edge of SBNTs synthesized at $700{ }^{\circ} \mathrm{C}$ is given in Figure 1c. It should be noted that around $130 \mathrm{eV}$ the P-L2,3-edge occurs which overlaps with the $\mathrm{Si}_{-} \mathrm{L}_{1}$-edge. In addition to the $\mathrm{Si}-\mathrm{L}_{2,3}$-edge, the ELNES of the P- $\mathrm{L}_{2,3^{-}}, \mathrm{N}-$ and O-Kedges measured at SBNTs prepared by a high $\mathrm{SiCl}_{4}$ precursor concentration do also not vary from those taken at samples synthesized with a low $\mathrm{SiCl}_{4}$ precursor concentration (Dennenwaldt et al., 2014).

The diameter and the length of the SBNTs can be tuned by varying the synthesis temperature. This phenomenon has been addressed in a former work but not further discussed until now (Sedlmaier et al., 2012). In the present work, the diameter was determined by averaging over several diameters of SBNTs depicted in TEM images which have been synthesized using low and high $\mathrm{SiCl}_{4}$ precursor concentrations. As the SBNTs exhibit a bamboo-like structure, it was averaged over the smaller and larger diameter leading to a relatively large deviation. Most SNTs described elsewhere exhibit a straight structure since they are synthesized with template-directing agents. Therefore, their diameter is constant and dependent on the template (García-Calzón and Díaz-García, 2012; Yang et al., 2011).

Figure $2 \mathrm{a}$ illustrates the dependency of the wall thickness and diameter on the temperature and $\mathrm{SiCl}_{4}$ precursor concentration. In the case of SBNTs synthesized with a low $\mathrm{SiCl}_{4}$ precursor concentration the wall thickness does not change with increasing temperature and remains constant at around $15 \mathrm{~nm}$. Also the diameter stays approximately constant $(120-130 \mathrm{~nm})$ for temperatures up to $500{ }^{\circ} \mathrm{C}$ however further up decreasing to about $70-80 \mathrm{~nm}$ (Figure 2a). The maximum length of the SBNTs seems to be rather constant to temperatures of up to $500{ }^{\circ} \mathrm{C}$ and values roughly around $2 \mu \mathrm{m}$ are determined by SEM investigations. For higher temperatures the maximum length increases strongly to more than 8 $\mu \mathrm{m}$ (see Supplemental Information Figure S1). For the SBNTs synthesized with high $\mathrm{SiCl}_{4}$ precursor concentration, the temperature dependency is different. The diameter also seems to be constant for low temperatures but then strongly increases above $500{ }^{\circ} \mathrm{C}$ as can be seen in Figure $2 \mathrm{a}$. Between $200{ }^{\circ} \mathrm{C}$ and $500{ }^{\circ} \mathrm{C}$ they exhibit a diameter of about $90-100 \mathrm{~nm}$, whereas almost double the value of $\sim 180-$ $190 \mathrm{~nm}$ at $700{ }^{\circ} \mathrm{C}$. A similar trend is observed for the wall thickness and length. The wall thickness 
increases from average values of around $20 \mathrm{~nm}$ to values of more than $60 \mathrm{~nm}$ for the highest synthesis temperature while the increase in length is from around roughly $1 \mu \mathrm{m}$ to $4 \mu \mathrm{m}$ at $700{ }^{\circ} \mathrm{C}$.

The results are visualized schematically in Figure $2 \mathrm{~b}$. It is found that for temperatures up to $500{ }^{\circ} \mathrm{C}$ the $\mathrm{SiCl}_{4}$ precursor concentration as well as the temperature only weakly affect the growth and SBNTs volume. The SBNTs grown at low $\mathrm{SiCl}_{4}$ precursor concentration possess for low temperatures a slighter larger diameter and length but the wall thickness is lower compared to those synthesized with a high $\mathrm{SiCl}_{4}$ precursor concentration. A different observation is made for higher temperatures where the SBNT dimensions are now strongly dependent on the initial $\mathrm{SiCl}_{4}$ precursor concentration. SBNTs grown with a low $\mathrm{SiCl}_{4}$ precursor at high temperatures are long and have a small diameter (the wall thickness remains) while the ones synthesized at low $\mathrm{SiCl}_{4}$ precursor concentration are short but possess a large diameter and wall thickness. A similar result has been reported for CNTs where higher synthesis temperature can also either lead to decreasing diameters and increasing length or the opposite, increasing diameters and decreasing length depending on the synthesis conditions (Aksak et al., 2009; Bronikowski, 2007). It is worth to mention that in contrast to most syntheses of CNT structures, no catalyst is necessary for the formation of SBNTs.

A possible explanation for the formation of the SBNTs could be based on Kirkendall effect (Anderson and Tracy, 2014; Fan et al., 2007). The Kirkendall effect links the diffusion of mass and vacancies to difference in atom concentration, i.e. local fluctuations could be responsible for the growth of the SBNTs and explain the branching. The starting materials, the two molecules $\mathrm{OP}\left(\mathrm{NH}_{2}\right)_{3}$ and $\mathrm{SP}\left(\mathrm{NH}_{2}\right)_{3}$, might provide the basic tetrahedral shape of the mesocrystal, i.e. the 3D assembly of the SBNTs. SEM images of the starting materials, which hint some tetrahedral shape, are provided in the Supplemental Information (Figure S2). In combination with the liquid $\mathrm{SiCl}_{4}$ precursor the SBNTs could form through diffusion in which $\mathrm{P}$ and $\mathrm{N}$ outdiffuse and $\mathrm{Si}$ and $\mathrm{O}$ indiffuse. The Kirkendall effect could induce the hollowing process of the starting materials resulting in the formation of SBNTs and their networks, respectively ( $\mathrm{Si}$ and $\mathrm{O}$ dominated) and flakes ( $\mathrm{P}$ and $\mathrm{N}$ dominated) as well as in the release of gases $\left(\mathrm{HCl}, \mathrm{H}_{2} \mathrm{~S}\right.$ and $\left.\mathrm{NH}_{3}\right)$. In the initial stage of the growth weakly condensed inorganic polymer agglomerations $\mathrm{Si} /(\mathrm{P}) / \mathrm{O} /(\mathrm{N})$ are formed which act as nuclei (Sedlmaier et al. 2012). It seems that for low temperatures this condensation reaction is only weakly influenced by the $\mathrm{SiCl}_{4}$ precursor concentration and restricted by the sluggish outward diffusion of $\mathrm{P}$ and $\mathrm{N}$. For temperatures higher than $500{ }^{\circ} \mathrm{C}$ the growth behavior changes and large nuclei are formed for the high $\mathrm{SiCl}_{4}$ precursor concentration possessing small curvatures and thus reduced diffusion at the tip of the growing SBNTs. In contrast, for the low $\mathrm{SiCl}_{4}$ precursor concentrations small nuclei are formed leading to SBNTs with large lengths and small diameter. The relationship between temperature and diameter is still ambiguous and has to be further investigated. Concerning the yield of the SBNTs further experiments were done by varying the $\mathrm{O}$ amount but no increase of SBNTs could be observed. The known structures, flakes and SBNTs could not been observed anymore. 
To characterize the SBNT mesocrystals two FIB lamellae of 3D assemblies synthesized with low $\mathrm{SiCl}_{4}$ precursor amount at temperatures of 300 and $700{ }^{\circ} \mathrm{C}$ were prepared. These networks were studied concerning structure, i.e. how they are built up and if they are hollow or filled. The chemical distribution of the elements $\mathrm{Si}, \mathrm{O}, \mathrm{N}$ and $\mathrm{P}$ in the SBNTs has been analyzed in previous works (Dennenwaldt et al., 2014; Sedlmaier et al., 2012). Figure 3a shows a secondary electron (SE) zoom-in image of a 3D assembly of the SBNTs synthesized at $700{ }^{\circ} \mathrm{C}$. A FIB cut through a mesocrystal is presented in Figure 3b. A higher magnification of the inside of the tetrahedral structure (Figure 3c) shows that the SBNTs are occurring homogeneously. The mesocrystals consist exclusively of SBNTs, i.e. neither a solid core or substrate which could be the starting point of the mesocrystal, nor a hole or cavity which could mean that all the starting material has been transformed into SBNTs, can be observed. TEM images of the lamellae prepared from the interior of the tetrahedral and synthesized at $300{ }^{\circ} \mathrm{C}$ and $700{ }^{\circ} \mathrm{C}$ confirm a well-ordered network.

To illuminate the internal morphology of the SBNTs mesocrystals, STEM-HAADF tomography in the TEM was performed. STEM-HAADF tomography experiments were done on a several $\mu \mathrm{m}$ large SBNTs network synthesized at $700{ }^{\circ} \mathrm{C}$ with a low amount of $\mathrm{SiCl}_{4}$ precursor. 70 images have been acquired from a tilt range of $\pm 70^{\circ}$ with $2^{\circ}$ tilt steps (3c). The samples did not show conspicuous changes after irradiation. The tilt series is shown in the Supplementary Information (S3). Still, an accurate reconstruction of the whole SBNT network was not possible due to drift during acquisition. Therefore, STEM-HAADF tomography was done on a small cluster of SBNTs synthesized at $700{ }^{\circ} \mathrm{C}$ with a low amount of $\mathrm{SiCl}_{4}$ precursor. A tilt-series was recorded at $80 \mathrm{kV}$ to avoid beam damage of the sample. The tilting range amounted $\pm 70^{\circ}$ with steps of $10^{\circ}$. STEM images of tilting angles of $-70^{\circ}$, $20^{\circ}, 10^{\circ}$ and $50^{\circ}$ are shown in the Supplementary Information (Figure S4). STEM-HAADF tomography reconstruction of a SBNTs assembly is shown in Figure 4, the average diameter of $70 \mathrm{~nm}$ (red arrows) of the SBNTs is in good agreement with the TEM results. The network consists of five SBNTs that are connected to each other. Thirteen STEM images were used for reconstructing the SBNTs assembly through a discrete tomography based on a masked simultaneous iterative reconstruction technique (SIRT) algorithm (Zürner et al., 2012). In this technique, an automated masking algorithm finds a rough 3D outline of the particle. This mask is further improved computationally and results in a reliable gray value for the material when applied to the SIRT algorithm. Hence, the second modified SIRT algorithm which gives a binary reconstruction yields very good results, tackling two major problems of tomographic reconstruction: porous structures and a strongly limited number of projections from beam-sensitive materials. However, some artifacts occur (red circles) due to beam damage limitation which affected the upper horizontal nanotube during acquisition. Apart from that, no altering of the SBNTs network structure could be observed. A cutthrough of different directions of the same fragment is shown in Error! Reference source not found. $4 \mathrm{~b}$ and $\mathrm{c}$. These images prove the hollow tubular morphology and clarify the bamboo-like structure. Due to the binary nature of the reconstruction, no manual threshold has to be set to determine surfaces. 
Thus, the inner diameter of the tubes at the junctions can be directly measured and results in openings of approximately $70 \mathrm{~nm}$. A movie of the 3D reconstruction is enclosed in the Supplementary Information (S5). It can clearly be seen that the junction points of the SBNTs are open and that they are connected to each other, which is of high importance for possible applications where filling of the SBNTs is required. The algorithm has successfully been applied to experimental images as shown here for the SBNTs. It allows the successful reconstruction of a tomogram using only a few images which is very useful for e.g. beam sensitive material where short acquisition times and a low accumulated dose are needed. All in all, this reconstruction technique offers huge potential to investigate materials that are otherwise easily destroyed during electron beam bombardment, e.g. biomolecules.

\section{Summary and conclusion}

$\mathrm{SiCl}_{4}$ precursor concentration during synthesis is a crucial factor to obtain nicely arranged $3 \mathrm{D}$ assemblies of SBNTs (Sedlmaier et al., 2012). Therefore, we increased the $\mathrm{SiCl}_{4}$ precursor content at different synthesis temperatures $\left(200-700{ }^{\circ} \mathrm{C}\right)$ while keeping all other reaction parameters constant. SBNTs and their mesocrystals as well as flakes and hollow particles were observed for every temperature. EDX and EELS measurements showed that the chemical composition does not change drastically compared to $\mathrm{SBNTs}$ synthesized with low $\mathrm{SiCl}_{4}$ precursor concentration. The elements are homogeneously distributed in the SBNTs. EELS measurements proved that Si is very likely to be tetrahedrally coordinated primarily with $\mathrm{O}$ and $\mathrm{N}$ atoms. However, the diameter of the SBNTs changes depending on the amount of $\mathrm{SiCl}_{4}$ precursor and the temperature. For low $\mathrm{SiCl}_{4}$ precursor concentration the diameter of SBNTs decreases at higher temperatures, while high $\mathrm{SiCl}_{4}$ precursor concentration leads to an increase with temperature. More well-ordered mesocrystals consisting of amorphous SBNTs were found at higher temperatures for both $\mathrm{SiCl}_{4}$ precursor concentrations. This is of importance in case the SBNTs are considered for applications, e.g. in nanofilters or nanofluidic systems. FIB sectioning, electron microscopy and STEM-HAADF tomography proved the hollow tubular structure of the 3D assembly of SBNTs. In addition, it could be shown that a reliable reconstruction can even be achieved by only thirteen STEM-HAADF images, using discrete tomography based on a SIRT algorithm, without altering the structure of the SBNTs assembly.

\section{References}

Agrawal, Y., Kedawat, G., Kumar, P., Dwivedi, J., Singh, V.N., Gupta, R.K., Gupta, B.K., 2015. High-performance stable field emission with ultralow turn on voltage from rGO conformal coated TiO2 nanotubes 3D arrays. Sci. Rep. 5, 11612. doi:10.1038/srep11612

Aksak, M., Kir, S., Selamet, Y., 2009. Effect of the growth temeprature on carbon nanotubes grown by thermal chemcial vapor deposition method. J. Optoelectron. Adv. Mater. 1, 281-284. 
Anderson, B.D., Tracy, J.B., 2014. Nanoparticle conversion chemistry: Kirkendall effect, galvanic exchange, and anion exchange. Nanoscale 6, 12195. doi:10.1039/c4nr02025a

Betzler, S.B., Wisnet, A., Breitbach, B., Mitterbauer, C., Weickert, J., Schmidt-Mende, L., Scheu, C., 2014. Template-free synthesis of novel, highly-ordered 3D hierarchical Nb $3 \mathrm{O} 7(\mathrm{OH})$ superstructures with semiconductive and photoactive properties. J. Mater. Chem. A 2, 12005. doi:10.1039/C4TA02202E

Bréchignac, C., Houdy, P., Lahmani, M., 2007. Nanomaterials and nanochemistry. Springer Publishing, Berlin, Heidelberg, New York.

Britt, D.K., Yoon, Y., Ercius, P., Ewers, T.D., Alivisatos, A.P., 2013. Hexameric octahedral clusters of $\mathrm{PbSe}$ nanocrystals grown from amorphous Lead(II) Carboxylate nanoparticles. Chem. Mater. 25, 2544-2548. doi:10.1021/cm401083g

Bronikowski, M.J., 2007. Longer nanotubes at lower temperatures: The influence of effective activation energies on carbon nanotube growth by thermal chemical vapor deposition. J. Phys. Chem. C 111, 17705-17712. doi:10.1021/jp071079y

Dennenwaldt, T., Sedlmaier, S.J., Binek, A., Schnick, W., Scheu, C., 2014. Bonding behavior and chemical stability of Silica-based nanotubes and their 3D assembly. J. Phys. Chem. C 118, 84168423. doi:10.1021/jp500408x

E. Biermans, L. Molina, K. J. Batenburg, S. Bals, G.V.T., 2010. Measuring porosity at the nanoscale by quantitative electron tomography. Nano Lett. 10, 5014-5019. doi:10.1021/nl103172r

Fan, H.J., Gösele, U., Zacharias, M., 2007. Formation of nanotubes and hollow nanoparticles based on Kirkendall and diffusion processes: A review. Small 3, 1660-1671. doi:10.1002/smll.200700382

Fan, Y., Ding, Y., Ma, H., Teramae, N., Sun, S., He, Y., 2015. Optical waveguide sensor based on silica nanotube arrays for label-free biosensing. Biosens. Bioelectron. 67, 230-236. doi:10.1016/j.bios.2014.08.021

García-Calzón, J.A., Díaz-García, M.E., 2012. Synthesis and analytical potential of silica nanotubes. Trends Anal. Chem. 35, 27-38. doi:10.1016/j.trac.2012.01.003

Hu, K.-W., Hsu, K.-C., Yeh, C.-S., 2010. pH-dependent biodegradable silica nanotubes derived from $\mathrm{Gd}(\mathrm{OH}) 3$ nanorods and their potential for oral drug delivery and MR imaging. Biomaterials 31 , 6843-8. doi:10.1016/j.biomaterials.2010.05.046

Hungría, A.B., Eder, D., Windle, A.H., Midgley, P.A., 2009. Visualization of the three-dimensional microstructure of $\mathrm{TiO} 2$ nanotubes by electron tomography. Catal. Today 143, 225-229. doi:10.1016/j.cattod.2008.09.014

Hyun, J.K., Ercius, P., Muller, D.A., 2008. Beam spreading and spatial resolution in thick organic specimens. Ultramicroscopy 109, 1-7. doi:10.1016/j.ultramic.2008.07.003 
Joshi, R.K., Schneider, J.J., 2012. Assembly of one dimensional inorganic nanostructures into functional 2D and 3D architectures. Synthesis, arrangement and functionality. Chem. Soc. Rev. 41, 5285. doi:10.1039/c2cs35089k

Klement, R., Koch, O., 1954. Phosphoroxy-triamid und Phosphorthio-triamid. Chem. Ber. 87, 333340.

Messaoudi, C., Boudier, T., Sanchez Sorzano, C.O., Marco, S., 2007. TomoJ: tomography software for three-dimensional reconstruction in transmission electron microscopy. BMC Bioinformatics 8, 288. doi:10.1186/1471-2105-8-288

Midgley, P.A., Dunin-Borkowski, R.E., 2009. Electron tomography and holography in materials science. Nat. Mater. 8, 271-280. doi:10.1038/nmat2406

Ni, B., Liu, H., Wang, P., He, J., Wang, X., 2015. General synthesis of inorganic single-walled nanotubes. Nat. Commun. 6, 8756. doi:10.1038/ncomms9756

Rao, C.N.R., Govindaraj, A., 2009. Synthesis of inorganic nanotubes. Adv. Mater. 21, 4208-4233. doi:10.1002/adma.200803720

Rasband, W.S., 1997-2011. ImageJ, U. S. National Institutes of Health, Bethesda, Maryland, USA.

Ren, L., Simmons, T.J., Lu, F., Rahmi, O., Kotha, S.P., 2014. Template free and large-scale fabrication of silica nanotubes with centrifugal jet spinning. Chem. Eng. J. 254, 39-45. doi:10.1016/j.cej.2014.05.077

Schnick, W., 1989. Synthese, Struktur und thermisches Verhalten von Thiophosphorsäuretriamid SP(NH2)3. Zeitschrift für Naturforsch. B 44b, 942-945.

Sedlmaier, S.J., Dennenwaldt, T., Scheu, C., Schnick, W., 2012. Template-free inorganic synthesis of silica-based nanotubes and their self-assembly to mesocrystals. J. Mater. Chem. 22, 15511. doi:10.1039/c2jm32100a

See Supplementary Information at [URL will be inserted] for images and movies of the SBNTs.

Sorzano, C.O.S., Messaoudi, C., Eibauer, M., Bilbao-Castro, J.R., Hegerl, R., Nickell, S., Marco, S., Carazo, J.M., 2009. Marker-free image registration of electron tomography tilt-series. BMC Bioinformatics 10, 124. doi:10.1186/1471-2105-10-124

Suh, Y.J., Lu, N., Park, S.Y., Lee, T.H., Lee, S.H., Cha, D.K., Lee, M.G., Huang, J., Kim, S.S., Sohn, B.H., Kim, G.H., Ko, M.J., Kim, J., Kim, M.J., 2013. Three-dimensional observation of TiO2 nanostructures by electron tomography. Micron 46, 35-42. doi:10.1016/j.micron.2012.12.002

Yang, X., Tang, H., Cao, K., Song, H., Sheng, W., Wu, Q., 2011. Templated-assisted one-dimensional silica nanotubes: synthesis and applications. J. Mater. Chem. 21, 6122-6135. doi:10.1039/c0jm04516k

Zürner, A., Döblinger, M., Cauda, V., Wei, R., Bein, T., 2012. Discrete tomography of demanding 
samples based on a modified SIRT algorithm. Ultramicroscopy 115, 41-49.

doi:10.1016/j.ultramic.2012.01.015

\section{Figure captions}

Figure 1. Sample synthesized at $700{ }^{\circ} \mathrm{C}$ with a high $\mathrm{SiCl}_{4}$ precursor content a) $\mathrm{SEM}$ image showing an overview of the specimen, the three reaction products (SBNTs, flakes, hollow particles) and the SBNTs 3D assemblies are marked. b) TEM image of SBNTs and c) comparison of the $\mathrm{Si}-\mathrm{L}_{2,3}$-edges of SBNTs synthesized with a low (green) and a high (red) concentration of $\mathrm{SiCl}_{4}$ precursor.

Figure 2. a) Diagram showing the dependence of diameter and wall thickness of the SBNTs on the synthesis temperature. For clarity reasons trend lines are added as doted lines. b) Growth model illustrating the influence of temperature and $\mathrm{SiCl}_{4}$ precursor concentration on the characteristic dimensions of the SBNTs. A low amount of $\mathrm{SiCl}_{4}$ precursor at low temperature leads to longer SBNTs at higher temperatures and a high amount of $\mathrm{SiCl}_{4}$ precursor at low temperature leads to SBNTs with a higher diameter at higher temperatures.

Figure 3. Sample synthesized at $700{ }^{\circ} \mathrm{C}$ with a low amount of $\mathrm{SiCl}_{4}$ precursor. a) zoom-in $\mathrm{SE}$ image of hyperbranched SBNTs, b) FIB cut through a SBNTs mesocrystal, the insert showing a higher magnification of the core of this 3D assembly and c) STEM image of a several $\mu \mathrm{m}$ broad SBNTs assembly synthesized at $700{ }^{\circ} \mathrm{C}$ with a low amount of $\mathrm{SiCl}_{4}$ precursor.

Figure 4. STEM-HAADF tomography reconstruction of a SBNTs assembly synthesized at $700{ }^{\circ} \mathrm{C}$ with a low amount of $\mathrm{SiCl}_{4}$ precursor showing a) calculated projection of the reconstructed NT network fragment, red circles showing artifacts, b) and c) cut through of the same fragment evincing that the SBNTs are hollow and that the junction points are open, red arrows denoting an approximate diameter of $70 \mathrm{~nm}$.

\section{Acknowledgement}

The authors gratefully acknowledge financial support that was granted by the DFG via the excellence cluster Nanosystems Initiative Munich (NIM) as well as from the Fonds der Chemischen Industrie (FCI). The authors acknowledge support of the National Center for Electron Microscopy, Lawrence Berkeley Lab, which is supported by the U.S. Department of Energy under Contract DE-AC0205CH11231. Additionally, we thank Christian Minke for the SEM investigations as well as Ramona 
Hoffmann and Sonja Matich for the FIB specimen preparation. Jim Ciston and Peter Ercius are gratefully acknowledged for the HAADF-TEM tilt series acquisition. 
Figure 1

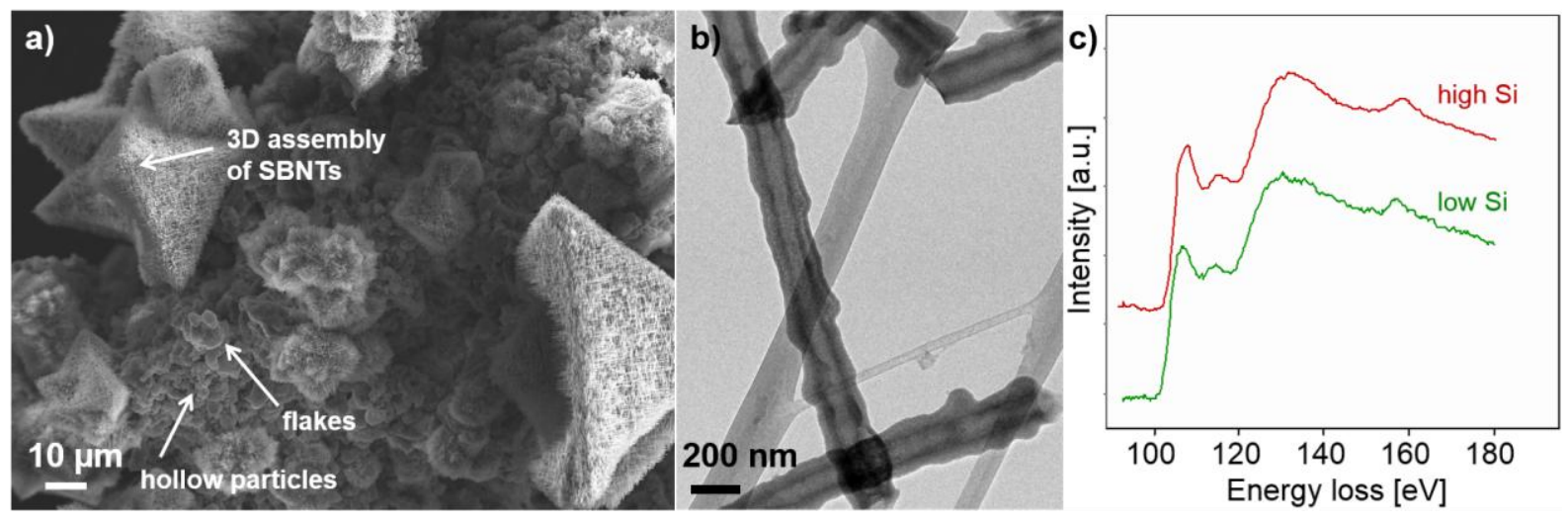


Figure 2

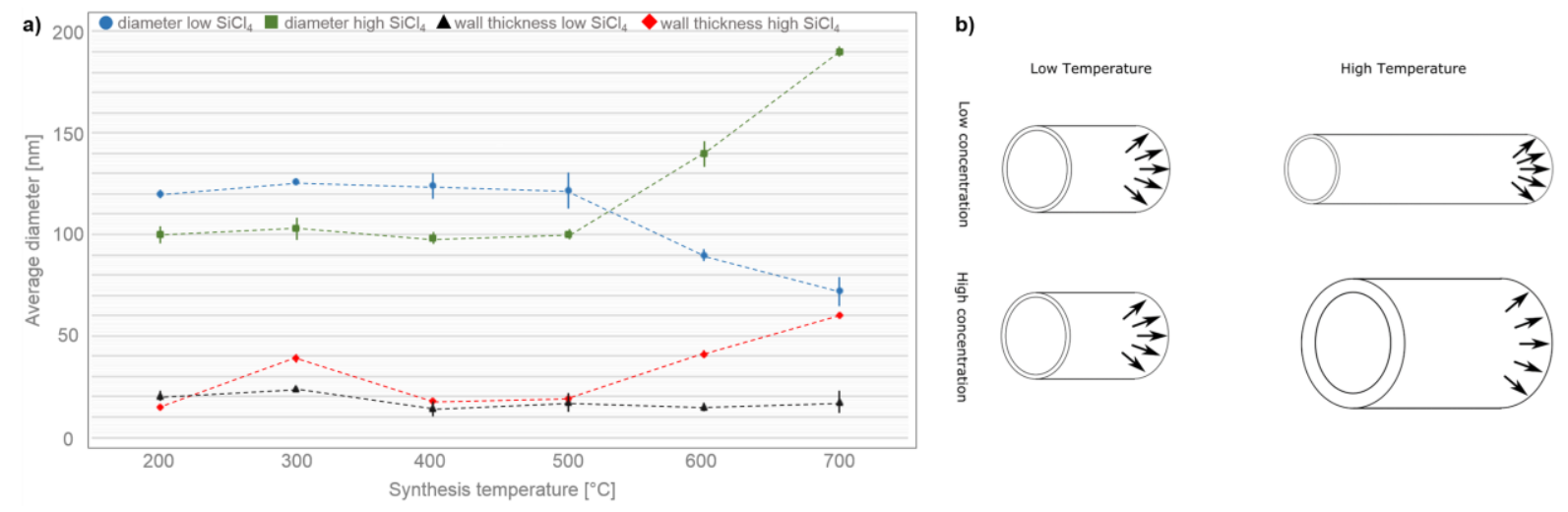

14 
Figure 3

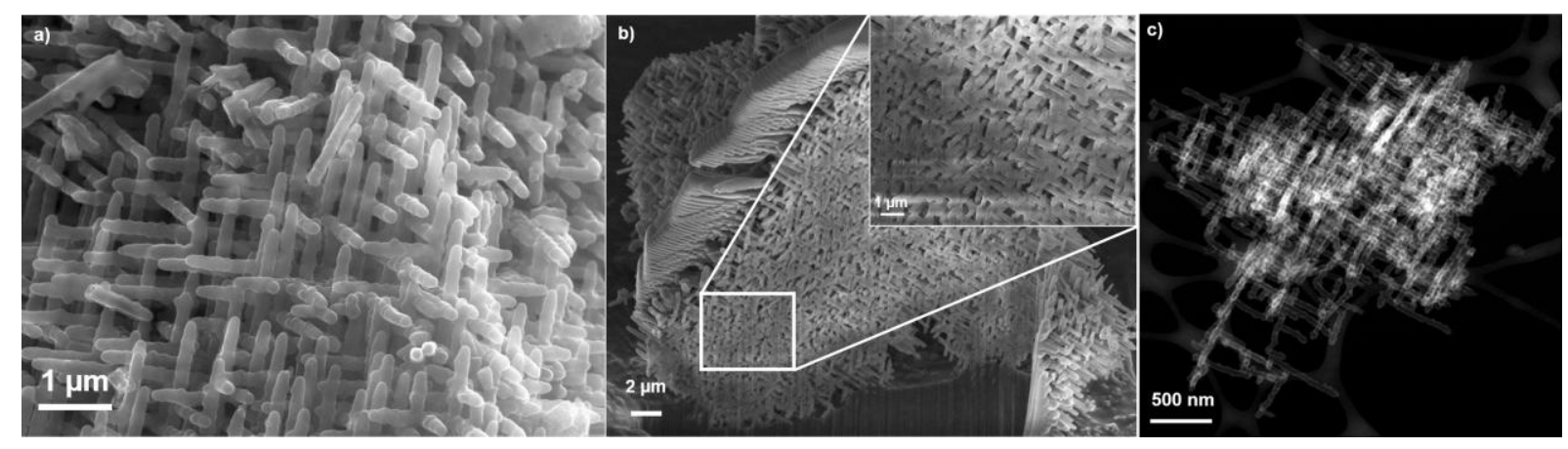




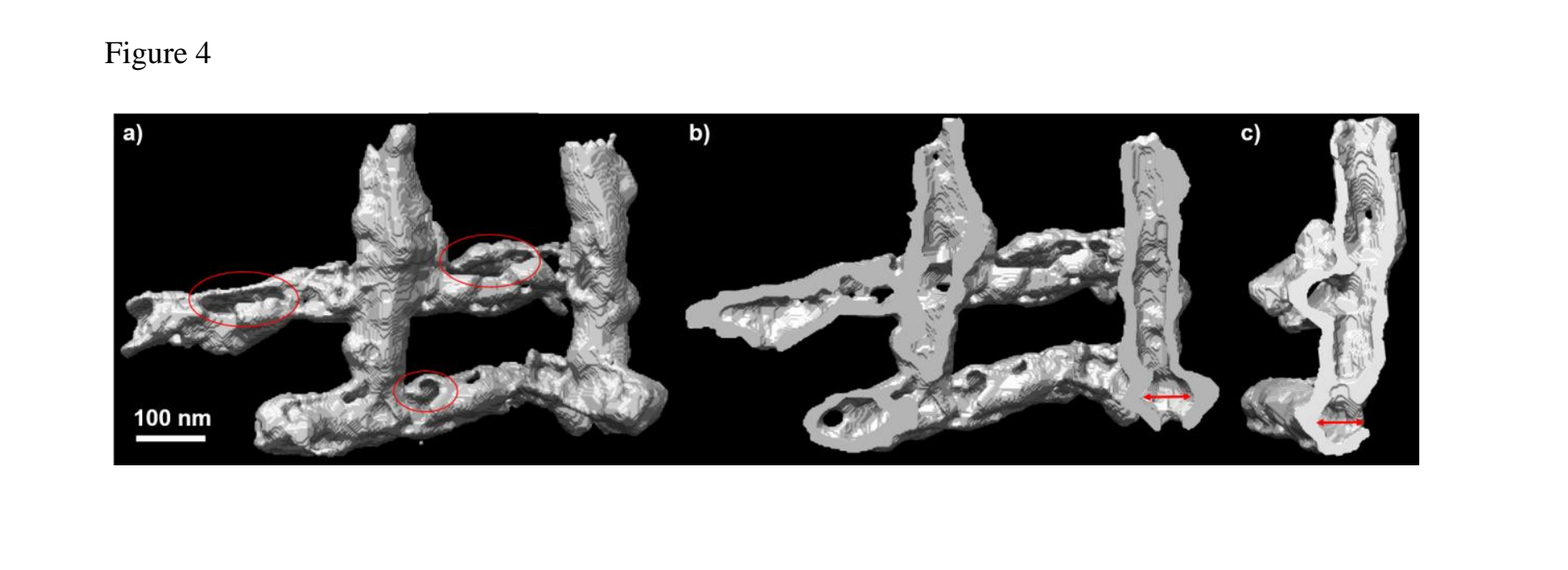

